\title{
BOUNDARY ELEMENT ANALYSIS OF THIN-WALLED STRUCTURES USING AN EXPANDING ELEMENT INTERPOLATION METHOD
}

\author{
JIANMING ZHANG, YUDONG ZHONG, LE YANG, BAOTAO CHI \& CHUANMING JU \\ State Key Laboratory of Advanced Design and Manufacturing for Vehicle Body, \\ College of Mechanical and Vehicle Engineering, Hunan University, China
}

\begin{abstract}
Boundary element analysis of thin-walled structures using a new expanding element interpolation method is presented. The element is obtained by adding virtual nodes based on a traditional discontinuous element. Coupled with the virtual nodes, the shape functions become higher order shape functions and are named fine shape functions. The original shape function of the discontinuous element is named raw shape function. With the expanding element, the interpolation accuracy can be increased by at least two orders compared with the original discontinuous element. The elements are able to naturally and accurately interpolate both continuous and discontinuous fields. The virtual nodes can be obtained by those of adjacent source points using the raw shape functions. Furthermore, the boundary integral equations are built up only at the inner nodes. Thus the size of the equations has not changed. Numerical examples are presented to verify our methods. Results demonstrate the accuracy and efficiency of the proposed method.
\end{abstract}

Keywords: boundary element method, expanding element, boundary integral equations.

\section{INTRODUCTION}

Accurate and efficient analysis of super thin structures is of crucial importance in engineering applications [1], [2]. The boundary element method (BEM) [3]-[9] has become recently an attractive alternative for problems in computational mechanics, and has been widely used to solve the problems of computational mechanics. Compared with the FEM, the BEM is a boundary method. BEM has evident advantages for solving problems such as linear elasticity [10], crack propagation [11], acoustics and contact problem [12], [13], and so on. In modern daily industrial settings, mesh preparation is the most labour intensive in numerical modelling, particularly for the FEM. The BEM needs not to deal with the interior mesh, so it is more effective in mesh preparation. In addition, the FEM requires the trial functions $C^{0}$-continuous, but the BEM does not require that. In addition, the BEM has the advantages of dimensionality reduction and high accuracy of calculation. In view of the above observations, we can know that the BEM is a more suitable method to analyse thin-super thin structures since the BEM just requires one to discretize the boundary and the stress can be accurately calculated. Then combined with the advantages of BEM, the super thin structure can be accurately approximated.

At present, there are mainly two kinds of implementation for the BEM [14], one is to use discontinuous element interpolation, and the other is to use continuous element interpolation. In the specific numerical realization process, the discontinuous element configures the node inside the element, which provides convenience for the realization process, which simplifies the system equation and reduces the difficulty of grid generation, but increases the calculation of the whole system equation. The continuous element directly arranges the points on the geometric vertices of the element. The number of nodes is less than the number of noncontinuous nodes under the same order, and the calculation amount is small. However, in some cases, there are still some difficulties in processing the corner points [15]. 
In this paper, a new expanding element is proposed to synthesize the discontinuous and continuous element. The proposed element has all the advantages of previous element and overcoming their disadvantages, improving the interpolation accuracy at least by two without changing the degrees of freedom of the equation. Finally, a universal method is proposed to solve the near-singular integral of the thin structure, that is, the integral element is continuously subdivided according to the position of the source point, and each time subdivided into two identical sub-elements. Through this method, the near-singular integrals in thin structures can be accurately calculated.

The structure of the paper is as follows. Section 2 is to introduce the proposed element interpolation method. In Section 3, the new numerical implementation of BIE is described. Section 4 gives some numerical examples. Section 5 is the conclusions.

\section{THE EXPANDING ELEMENT INTERPOLATION METHOD}

\subsection{The expanding elements}

The expanding element is obtained by adding virtual nodes on the traditional discontinuous element. These elements have two kinds of shape functions: raw and fine shape function. As shown in Fig. 1, the two shape functions of the constant expanding element are as follows:

$$
\begin{gathered}
N^{r}=1, \\
\left\{\begin{array}{l}
N_{1}^{f}=-0.5 \xi(1-\xi) \\
N_{2}^{f}=(1-\xi)(1+\xi) \\
N_{3}^{f}=0.5 \xi(1+\xi),
\end{array}\right.
\end{gathered}
$$

where $N^{r}, N_{1}^{f}, N_{2}^{f}$ and $N_{3}^{f}$ denote the raw and fine shape function, respectively.

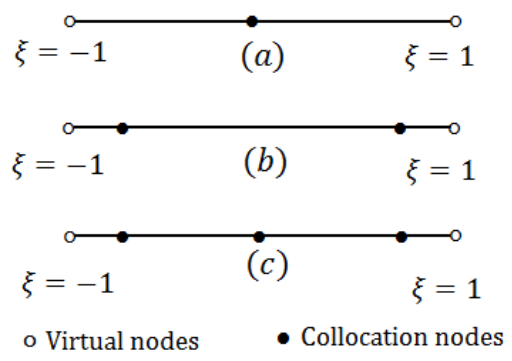

Figure 1: Constant, linear and quadratic expanding element.

\subsection{Introduction of the new interpolation method}

Firstly, we will dentally introduce the method of the calculation of the values of the virtual nodes (considering the example in Fig. 2).

When the expanding element is used to interpolate the known boundary conditions, the value of the virtual node is directly equal to the boundary conditions. When the expanding element is used to interpolate unknown boundary conditions, the node values of the virtual nodes can be obtained by the extrapolation of the raw shape functions. 


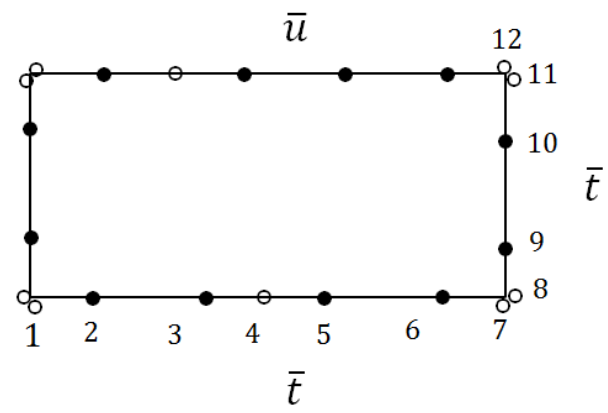

Figure 2: The geometric model for different boundary variables.

When the proposed method is used to interpolate the discontinuous boundary variables, we collocate two virtual nodes at a vertex shared by two adjacent elements. The nodal values of virtual nodes are calculated by eqn (3)

$$
\begin{aligned}
& \mathbf{t}_{7}=\overline{\mathbf{t}}_{7}, \mathbf{t}_{8}=\overline{\mathbf{t}}_{8} \\
& \mathbf{t}_{11}=\overline{\mathbf{t}}_{11}, \mathbf{t}_{12}=\overline{\mathbf{t}}_{12} .
\end{aligned}
$$

In this way, the discontinuous boundary conditions can be kept accurate. And for continuous fields (displacement boundary conditions), eqns (4) and (5) can be used

$$
\begin{gathered}
\mathbf{u}_{11}=\mathbf{u}_{12}=\overline{\mathbf{u}}_{11}, \\
\mathbf{u}_{7}=\mathbf{u}_{8}=\frac{1}{2}\left(N_{5}^{r}(1) \mathbf{u}_{5}+N_{6}^{r}(1) \mathbf{u}_{6}+N_{9}^{r}(-1) \mathbf{u}_{9}+N_{10}^{r}(-1) \mathbf{u}_{10}\right) .
\end{gathered}
$$

After getting the values of virtual node, the physical variables in the BIE can be obtained by interpolating by the fine shape functions. So the $\mathbf{u}$ and $\mathbf{t}$ on the right edge can be obtained by

$$
\begin{aligned}
& \mathbf{u}=N_{8}^{f} \mathbf{u}_{8}+N_{9}^{f} \mathbf{u}_{9}+N_{10}^{f} \mathbf{u}_{10}+N_{11}^{f} \mathbf{u}_{11} \\
& \mathbf{t}=N_{8}^{f} \mathbf{t}_{8}+N_{9}^{f} \mathbf{t}_{9}+N_{10}^{f} \mathbf{t}_{10}+N_{11}^{f} \mathbf{t}_{11} .
\end{aligned}
$$

Eqn (6) can be further written as

$$
\begin{aligned}
\mathbf{u}= & \frac{1}{2} N_{8}^{f} N_{5}^{r} \mathbf{u}_{5}+\frac{1}{2} N_{8}^{f} N_{6}^{r} \mathbf{u}_{6}+N_{11}^{f} \overline{\mathbf{u}}_{11} \\
& +\left(N_{9}^{f}+\frac{1}{2} N_{8}^{f} N_{9}^{r}\right) \mathbf{u}_{9}+\left(N_{10}^{f}+\frac{1}{2} N_{8}^{f} N_{10}^{r}\right) \mathbf{u}_{10} \\
\mathbf{t}= & N_{8}^{f} \overline{\mathbf{t}}_{8}+N_{9}^{f} \overline{\mathbf{t}}_{9}+N_{10}^{f} \overline{\mathbf{t}}_{10}+N_{11}^{f} \overline{\mathbf{t}}_{11} .
\end{aligned}
$$

From above introduction, when the boundary condition is known, the value of the virtual node is directly equal to the boundary condition or directly obtained by extrapolation of the raw shape functions. As the degrees of freedom of the virtual nodes need to be eliminated by the source points, thus and the size of the matrix equation does not change. Moreover, no 
matter discontinuous and continuity boundary variables, the expanding elements can also approximate accurately.

\section{NUMERICAL IMPLEMENTATION OF BIE}

\subsection{Nearly singular integration}

Accurate and efficient calculation of nearly singular integrals is the key to numerical calculations. In this part, we proposed an adaptive element subdivision method to solve nearly singular integrals. First we need to calculate the side length $l$ of the integral element, and then calculate the distance $d$ from the source point to the center of the element. In the global coordinate system, when $l$ is less than $d$, the integral of the element is a regular integral. Otherwise, the element is divided into two equal sub-elements (see Fig. 3). By using this method, the nearly singular integral can be accurately calculated, and no integral transformation is needed, and that is easily to implement.

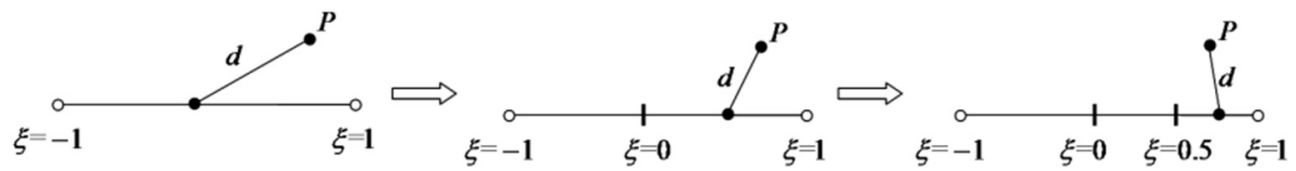

Figure 3: The method of element subdivision.

\subsection{Numerical implementation}

The well-known BIE for the solution of 2D linear elasticity problem in a bounded by $\Gamma$ is

$$
c(P) u(P)+\int_{\Gamma} q^{*}(P, Q) u(P) d \Gamma(Q)=\int_{\Gamma} u^{*}(P, Q) q(P) d \Gamma(Q),
$$

where $P$ and $Q$ are the source and the field point, respectively. The Kelvin fundamental solutions $u_{i j}^{*}(P, Q)$ and $t_{i j}^{*}(P, Q)$ are given by:

$$
\begin{gathered}
u_{i j}^{*}(P, Q)=\frac{1}{8 \pi \mu(1-v)}\left[(3-4 v) \delta_{i j} \ln \frac{1}{r}+r_{, i} r_{, j}\right], \\
t_{i j}^{*}(P, Q)=-\frac{1}{4 \pi(1-v) r}\left\{\frac{\partial r}{\partial n}\left[(1-2 v) \delta_{i j}+2 r_{, i} r_{, j}\right]-(1-2 v)\left(r_{, i} n_{j}-r_{, j} n_{i}\right)\right\},
\end{gathered}
$$

where $\mu$ and $v$ are the shear modulus and the Poisson's ratio, respectively. $r$ is the distance between the source and field point.

Eqn (8) is discretized with $N$ expanding elements. The discretized equations for BIE can be obtained

$$
c(P) u^{j}(P)+\sum_{i=1}^{N}\left(\int_{\Gamma_{i}} q^{*}(P, Q) \mathbf{N}^{f} d \Gamma(Q)\right) \mathbf{u}^{i}=\sum_{i=1}^{N}\left(\int_{\Gamma_{i}} u^{*}(P, Q) \mathbf{N}^{f} d \Gamma(Q)\right) \mathbf{t}^{i} .
$$

Eqn (11) can be expressed in a matrix form as

$$
\mathbf{H u}=\mathbf{G t},
$$


where vectors $\mathbf{u}$ and $\mathbf{t}$ consist of all nodal displacement and traction. $\mathbf{H}$ and $\mathbf{G}$ are shown as follows:

$$
\begin{aligned}
& \mathbf{H}_{i j}=\int_{\Gamma_{e}} \mathbf{t}^{*}\left(P^{i}, Q\right) N_{j}^{f}(Q) d \Gamma(Q), \\
& \mathbf{G}_{i j}=\int_{\Gamma} \mathbf{u}^{*}\left(P^{i}, Q\right) N_{j}^{f}(Q) d \Gamma(Q) .
\end{aligned}
$$

Distinguishing the different boundary conditions, eqn (12) can be further written as

$$
\left[\begin{array}{llll}
\overline{\mathbf{H}}^{s} & \mathbf{H}^{s} & \overline{\mathbf{H}}^{v} & \mathbf{H}^{v}
\end{array}\right]\left[\begin{array}{c}
\overline{\mathbf{u}}^{s} \\
\mathbf{u}^{s} \\
\overline{\mathbf{u}}^{v} \\
\mathbf{u}^{v}
\end{array}\right]=\left[\begin{array}{llll}
\mathbf{G}^{s} & \overline{\mathbf{G}}^{s} & \mathbf{G}^{v} & \overline{\mathbf{G}}^{v}
\end{array}\right]\left[\begin{array}{c}
\mathbf{t}^{s} \\
\overline{\mathbf{t}}^{s} \\
\mathbf{t}^{v} \\
\overline{\mathbf{t}}^{v}
\end{array}\right],
$$

where $\overline{\mathbf{u}}^{s}, \overline{\mathbf{t}}^{s}, \overline{\mathbf{u}}^{v}, \overline{\mathbf{t}}^{v}$ and $\mathbf{u}^{s}, \mathbf{t}^{s}, \mathbf{u}^{v}, \mathbf{t}^{v}$ are the known and unknown boundary variables of the source points and virtual points, respectively.

From 2.2, we can see that the relationship of source points and virtual points are as follows:

$$
\begin{aligned}
\mathbf{u}^{v} & =\mathbf{N}_{1}^{r} \mathbf{u}^{s} \\
\mathbf{q}^{v} & =\mathbf{N}_{2}^{r} \mathbf{q}^{s} .
\end{aligned}
$$

Using eqn (16), eqn (15) can be written as

$$
\mathbf{A x}=\mathbf{f}
$$

where

$$
\begin{aligned}
& \mathbf{A}=\left[\begin{array}{ll}
\mathbf{H}^{s}+\mathbf{H}^{v} \mathbf{N}_{r 1} & -\left(\mathbf{G}^{s}+\mathbf{G}^{v} \mathbf{N}_{r 2}\right)
\end{array}\right], \\
& \mathbf{x}=\left[\begin{array}{l}
\mathbf{u}^{s} \\
\mathbf{q}^{s}
\end{array}\right], \\
& \mathbf{f}=\left[\overline{\mathbf{G}}^{s} \overline{\mathbf{q}}^{s}+\overline{\mathbf{G}}^{v} \overline{\mathbf{q}}^{v}-\overline{\mathbf{H}}^{s} \overline{\mathbf{u}}^{s}-\overline{\mathbf{H}}^{v} \overline{\mathbf{u}}^{v}\right] .
\end{aligned}
$$

Matrix $\mathbf{A}$ is a square matrix of order $n ; \mathbf{x}$ is unknown at the source points; $\mathbf{f}$ is the known vector on the right-hand side.

From eqns (17)-(20), one can see that the degrees of freedom of the virtual nodes are eliminated by the source points, so the size of the system equation does not change. This point is the main advantage of the proposed method.

\section{NUMERICAL EXAMPLES}

To verify the advantages of the proposed method for thin-walled structures, a few illustrative numerical results are given in this section. In the following examples, e represents relative errors and $\mathrm{n}$ stands for the number of the nodes

$$
e=\frac{1}{|u|_{\max }} \sqrt{\frac{1}{N} \sum_{i=1}^{N}\left(u_{i}^{\text {num }}-u_{i}^{\text {exact }}\right)} .
$$


In all figures, ExpdConst, ExpdLinear, ExpdQuad, TradConst, TradLinear, and TradQuad represent the proposed expanding elements and traditional continuous elements (constant, linear, quadratic elements) interpolation methods with different orders, respectively.

\subsection{Example 1: Concentric circles subject to a displacement field problem}

In this case, the proposed method is used to analyze a thin coating on a shaft, and the geometry model is as shown in Fig. 4 (these radii are $r_{1}$ and $r_{2}$, respectively). The thickness of coating is $\mathrm{h}$ (varies in the range of $10^{-1} r_{1}-10^{-6} r_{1}$ ). It is subject to the boundary conditions of eqn (22). Plane strain case with $E=1, v=0.25$ (in consistent units). 60 nodes are used on each boundary. The relative errors and convergence rates of $t_{y}$ are shown in Figs 5 and 6.

$$
u_{x}=y^{3}-3 y x^{2}, u_{y}=-x^{3}+3 x y^{2} .
$$

It can be seen from Figs 5 and 6, when the thickness of the coating becomes smaller, the results by using the traditional element interpolation method are not correct, and that is difficult to improve the accuracy with too many nodes. Overall, when the coating is gradually thinned, the accuracy of using the proposed method is significantly higher and the convergence is better than the conventional discontinuous element interpolation method.

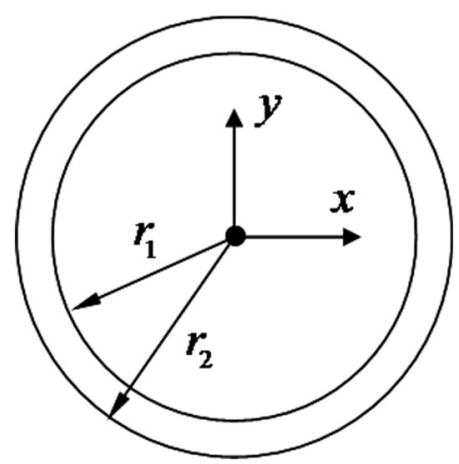

Figure 4: The simplified geometry model.

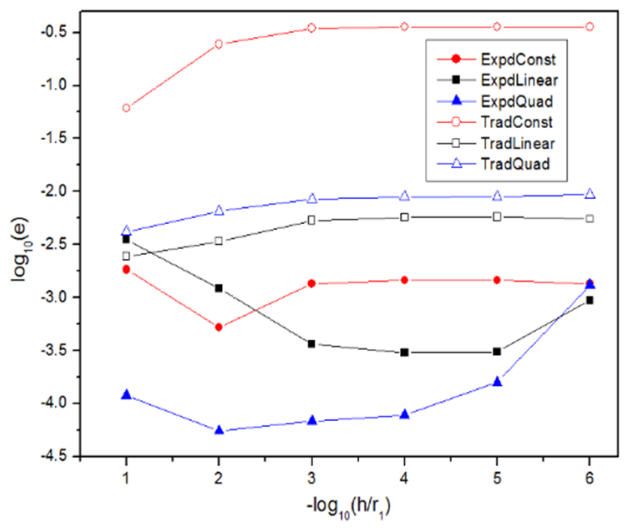

Figure 5: The relative errors of $t_{y}$ on the boundary of the inner circle. 


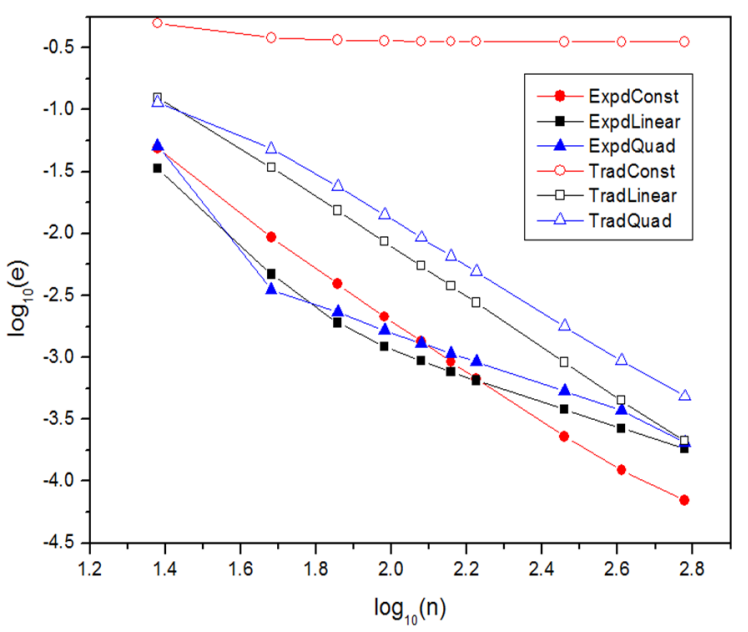

Figure 6: The convergence of $t_{y}$ on the boundary of the inner circle for $h / r_{l}=10-6$.

\subsection{Example 2: cantilever beam}

The expanding element interpolation method is also studied in the cantilever beam problem. Consider a beam of length $L=10$ and 100, respectively, height $h=1, E=1.1 \times 10^{5}, v=0.25$, subjected to a parabolic traction at the free end with $P(y)=1.0$ (as shown in Fig. 7). The analytical solution of the displacement is given by

$$
\begin{aligned}
& u_{x}=-\frac{P}{6 \bar{E} I}\left(y-\frac{h}{2}\right)\left[(6 L-3 x) x+(2+\bar{v})\left(y^{2}-2 h y\right)\right] \\
& u_{y}=\frac{P}{6 \bar{E} I}\left[3 \bar{v}\left(y^{2}-2 h y+\frac{1}{2} h^{2}\right)(L-x)+\frac{1}{4}(4+5 \bar{v}) h^{2} x+\left(L-\frac{1}{3} x\right) 3 x^{2}\right],
\end{aligned}
$$

where

$$
I=\frac{h^{3}}{12}, \quad \bar{E}=\frac{E}{1-v^{2}}, \quad \bar{v}=\frac{v}{1-v^{2}}
$$

and 50 elements are used on edge $a b$ and $c d$, respectively. On edge $a d$ and $b c$, we collocate only one element. The results of $u_{y}$ on the edge $a b$, together with the analytical solution are shown in Fig. 8. To study the accuracy and convergence rate, a series number of nodes are used to research that and the results are shown in Fig. 9.

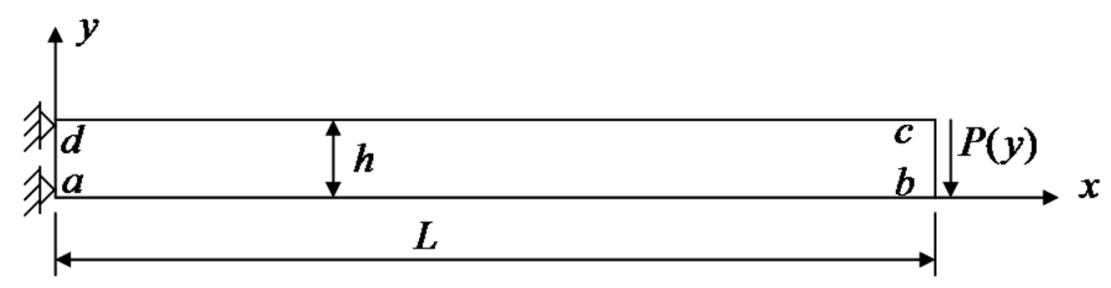

Figure 7: Cantilever beam problem. 


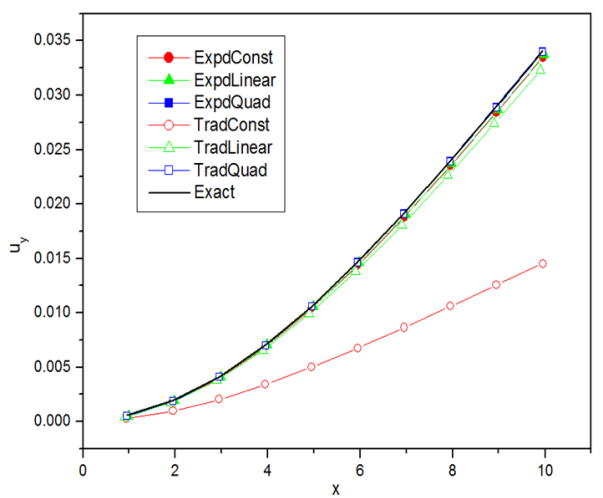

(a)

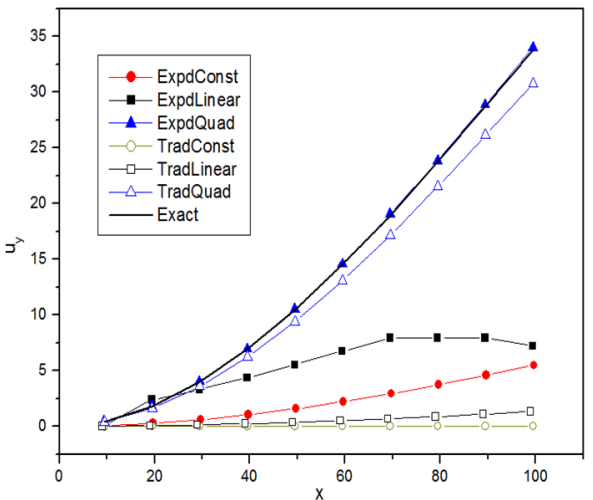

(b)

Figure 8: The $u_{y}$ along the edge $a b$. (a) $\mathrm{L}=10$; (b) $\mathrm{L}=100$.

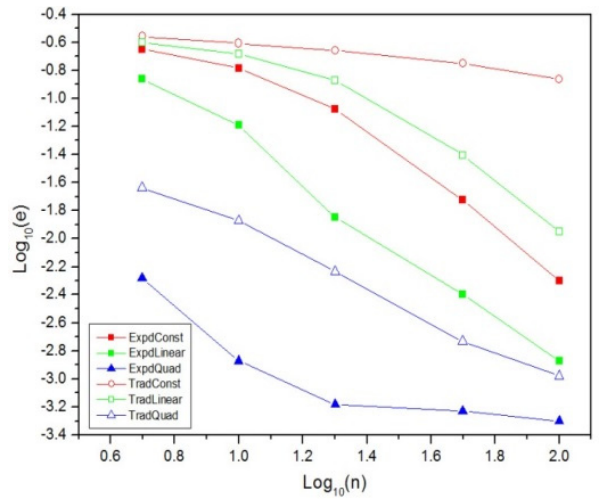

(a)

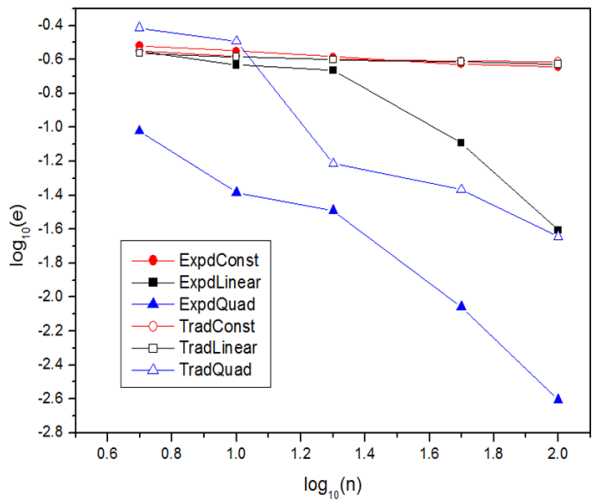

(b)

Figure 9: Convergence of $u_{y}$ on the edge $a b$. (a) $\mathrm{L}=10$; (b) $\mathrm{L}=100$.

From Figs 8 and 9, one can see that the accuracy and astringency rate by proposed method are higher than those by traditional elements interpolation method. The advantages of the proposed method are demonstrated again.

\subsection{Example 3: structures with negative Poisson's ratio}

To verify the universality of the proposed method, in this example, the proposed method is used to analysis a negative Poisson's ratio structure. Plane strain case with $P=1 \mathrm{Mpa}$, $E=1,000 \mathrm{MPa}$, Poisson's ratio $v=0.25$. The corresponding geometric parameters are given as follows: structure length $l=90$, height $h=60, t=2$ as shown in Fig. 10.

"ExpdQuad684" and "FEM1650" in Fig. 11 represent to use 684 and 1,650 nodes with different methods, respectively. The result of $u_{y}$ on the edge $a$ are as shown in Fig. 11. The result of 3,232,854 nodes by the FEM is taken as the reference solution. The von Mises stress by the proposed method and the FEM are as shown in Figs 12 and 13, respectively. 


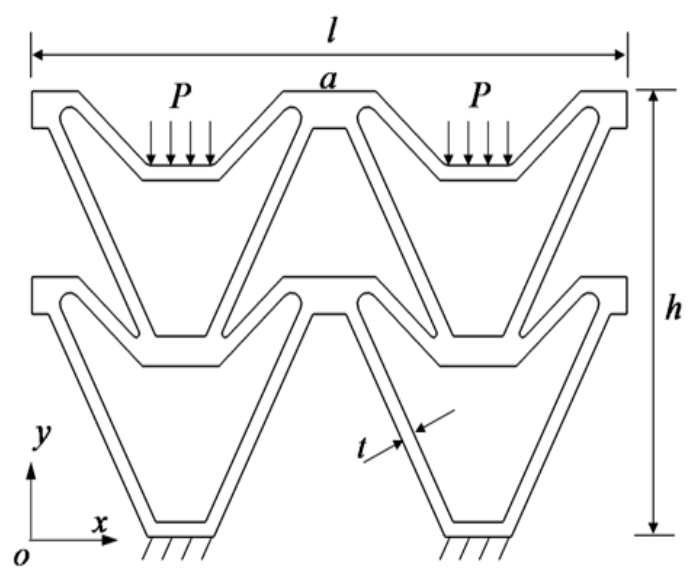

Figure 10: The geometric model of the structures.

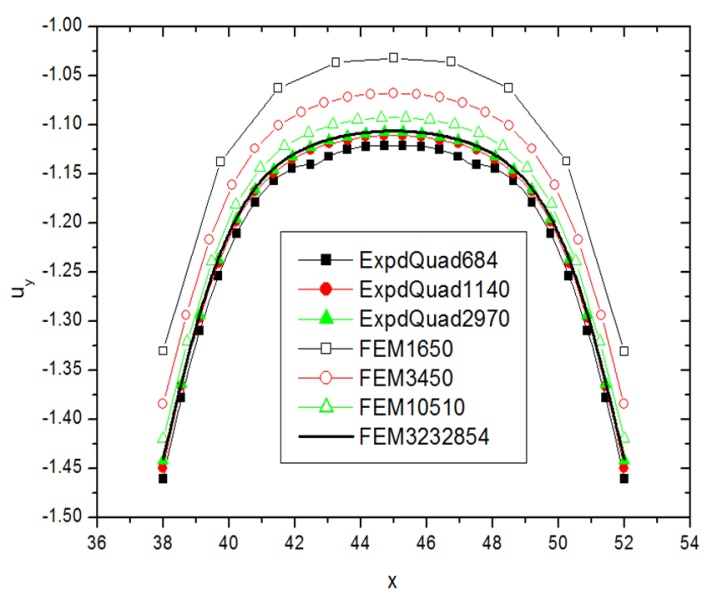

Figure 11: $u_{y}$ on the edge $a$.

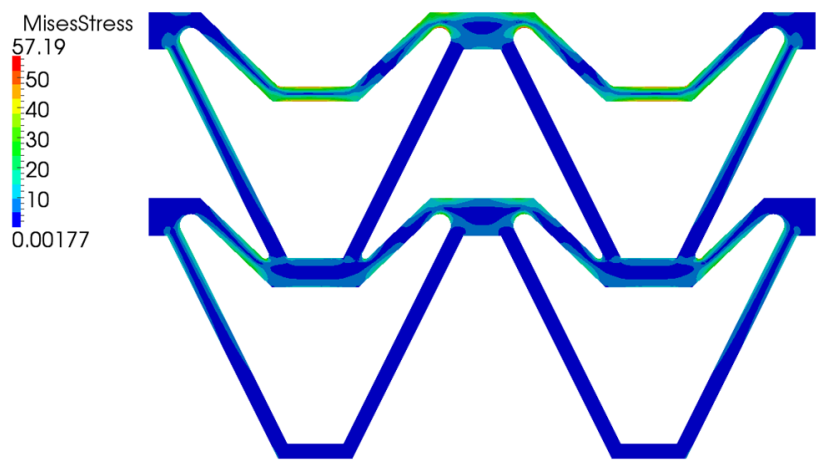

Figure 12: The result by using the proposed method with 1,140 nodes. 


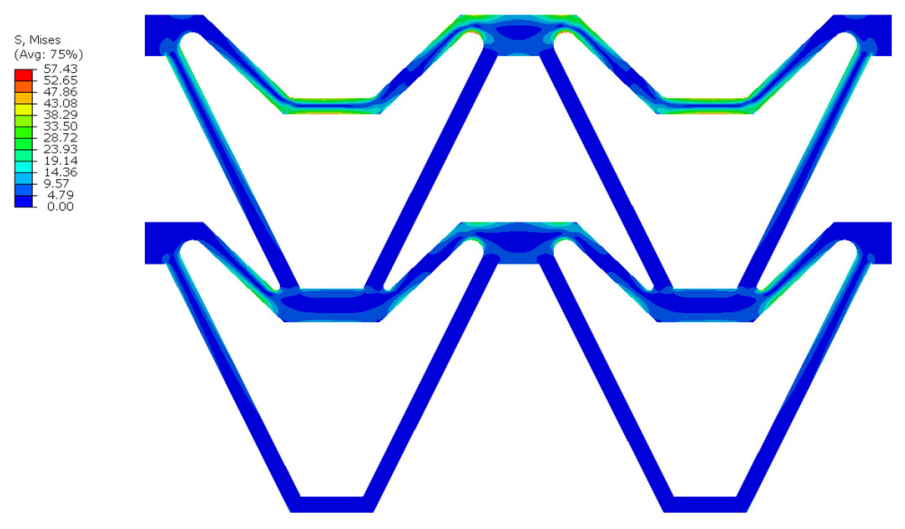

Figure 13: The result by using the FEM with 3,232,854 nodes.

\section{CONCLUSIONS}

This paper has presented a boundary element analysis of super thin structures using the expanding element interpolation method. Compared with FEM, there is no need for any assumptions in the BEM, and high accuracy can be obtained without using large numbers of elements. The proposed method not only has all the merits of the discontinuous element, but also guarantees the interelement continuity of field variables. Both continuous and discontinuous fields can be accurately interpolated by the proposed method. Moreover, as the degrees of freedom of the virtual nodes need to be eliminated, the size of the matrix equations does not increase. Results demonstrate that the proposed method possesses satisfactory accuracy and convergence rate compared with the traditional method. Extending our method to solve three-dimensional problems is ongoing.

\section{ACKNOWLEDGEMENT}

The research was supported by National Science Foundation of China (Grant No. 11772125).

\section{REFERENCES}

[1] Wempner, G., Mechanics and finite elements of shells. Applied Mechanics Reviews, 42(5), pp. 129-142, 1989.

[2] Yao, Z.H., A new type of high-accuracy BEM and local stress analysis of real beam, plate and shell structures. Engineering Analysis with Boundary Elements, 65, pp. 117, 2016.

[3] Zhang, J.M., Han, L., Lin, W.C., Dong, Y.Q. \& Ju, C.M., A new implementation of BEM by an expanding element interpolation method. Engineering Analysis with Boundary Elements, 78, pp. 1-7, 2017.

[4] Tanaka, M., Zhang, J.M. \& Matsumoto, T., Boundary-type meshless solution of potential problems: Comparison between singular and regular formulations in hybrid BNM Transactions of JASCOME. Journal of Boundary Element Methods, 20, pp. 2126, 2003.

[5] Zhou, F.L., Zhang, J.M., Sheng, X.M. \& Li, G.Y., A dual reciprocity boundary face method for 3D non-homogeneous elasticity problems. Engineering Analysis with Boundary Elements, 36, pp. 1301-1310, 2012. 
[6] Zhang, J.M., Qin, X.Y., Han, X. \& Li, G.Y., A boundary face method for potential problems in three dimensions. International Journal for Numerical Methods in Engineering, 80(3), pp. 320-337, 2009.

[7] Xie, G.Z., Zhang, J.M., Dong, Y.Q., Huang, C. \& Li, G.Y., An improved exponential transformation for nearly singular boundary element integrals in elasticity problems. International Journal of Solids and Structures, 51(6), pp. 1322-1329, 2013.

[8] Luo, J.F., Liu, Y.J. \& Berger, E.J., Analysis of two-dimensional thin structures (from micro- to nano-scales) using the boundary element method. Computational Mechanics, 22(5), pp. 404-412, 1998.

[9] Liu, Y.J., Analysis of shell-like structures by the boundary element method based on 3-D elasticity: Formulation and verification. International Journal for Numerical Methods in Engineering, 41(3), pp. 541-558, 1998.

[10] Manolis, G.D. \& Banerjee, P.K., Conforming versus non-conforming boundary elements in three-dimensional elastostatics. International Journal for Numerical Methods in Engineering, 23(10), pp. 1885-1904, 1986.

[11] Xie, G.Z., Zhang, J.M., Huang, C., Lu, C.J. \& Li, G.Y., A direct traction boundary integral equation method for three-dimension crack problems in infinite and finite domains. Computational Mechanics, 53, pp. 575-586, 2014.

[12] Soares, Jr. D. \& Mansur, W.J., An efficient stabilized boundary element formulation for 2D time-domain acoustics and elastodynamics. Computational Mechanics, 40, pp. 355-365, 2007.

[13] Blázquez, A., París, F. \& Mantič, V., BEM solution of two-dimensional contact problems by weak application of contact conditions with non-conforming discretizations. International Journal of Solids and Structures, 35, pp. 3259-3278, 1998.

[14] Parreira, P., On the accuracy of continuous and discontinuous boundary elements. Engineering Analysis, 5(4), pp. 205-211, 1988.

[15] Mukhopadhyay, S. \& Majumdar, N., A study of three-dimensional edge and corner problems using the neBEM solver. Engineering Analysis with Boundary Elements, 33(2), pp. 105-119, 2009. 